\title{
Two examples of partitioning approaches for multiscale and multiphysics coupled problems
}

\author{
David Dureisseix \\ Laboratoire de Mécanique et Génie Civil (LMGC) \\ CNRS UMR 5508/Université Montpellier 2 \\ CC048 Place Eugène Bataillon \\ F-34095 Montpellier cedex 5 \\ David.Dureisseix@lmgc.univ-montp2.fr
}

ABSTRACT. Partitioning strategies usually focus on interaction between subsystems. They are good candidates to model couplings at different spatial or time scales. A mechanism for the information transfer between subsystems and / or between scales is therefore required. Herein, we propose examples of a subdomain gluing with different spatial scales, and of a poroelastic coupling featuring different time scales.

RÉSUMÉ. Les techniques de partitionnement, qui mettent en avant les interactions entre soussystèmes, sont de bonnes candidates pour modéliser les couplages, en particulier en présence d'échelles spatiales ou temporelles différentes. Un mécanisme de transfert d'informations entre ces sous-systèmes, ou ces modélisations, est alors nécessaire. Nous présentons ici des exemples, tout d'abord pour un couplage entre échelles spatiales différentes, décrit dans le cadre d'une décomposition de domaine, puis pour un couplage poroélastique avec la prise en compte d'échelles temporelles différentes.

KEYWORDS: partitioning, interface, multiphysics, multiscale, transfer, non matching discretizations, finite elements.

MOTS-CLÉS : partitionnement, interface, multiphysique, multiéchelle, transfert, discrétisations incompatibles, éléments finis.

DOI:10.3166/REMN.17.807-818 @ 2008 Lavoisier, Paris 


\section{Introduction}

Improvement of the fidelity and prediction ability of numerical models often leads to an increase in simulation accuracy (and problem size), complexity of the constitutive relations (number of internal variables), or to take into account couplings between several physics (number of involved fields).

The simulation of such models requires the development of suited computational strategies. Amongst others, partitioning methods offer a large flexibility, an improved code durability, and an easier maintenance and evolution (for instance with code coupling). The partitioning can be geometric, for instance with domain decomposition, or between physics, for instance for fluid / structure interaction problems. These approaches focus on interactions between subsystems and often lead to isolate their treatment from the behavior of the subsystems themselves for which adequate techniques can be used in a modular fashion.

Usually, the reference coupled problem exhibits several scales (spatial or temporal ones) in the solution. A partitioning technique may take this fact into account for improving its efficiency or its practical implementation. Herein, we discuss two examples falling into this category: (i) the coupling between subdomains with different spatial scales, (ii) the coupling between different physics, with different time scales.

\section{Gluing spatial multiscale fields with domain decomposition}

The spatial geometric partitioning arising from a non overlapping domain decomposition naturally induces interfaces (surfaces for 3D problems) between subdomains (Le Tallec, 1994; Farhat et al., 1994; Magoulès, 2007), Figure 1 (a). If different spatial scales are present in the searched solution field, a dedicated multiscale representation of the field trace on the interfaces can be built. This can be used for several goals such as: homogenization of a subdomain, convergence acceleration of the domain decomposition algorithm, coupling different subdomains with different discretizations, etc.

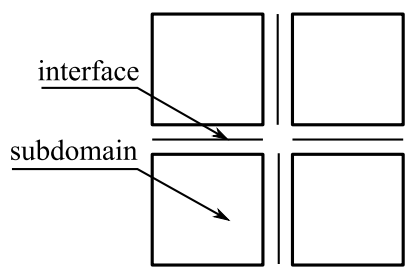

(a)

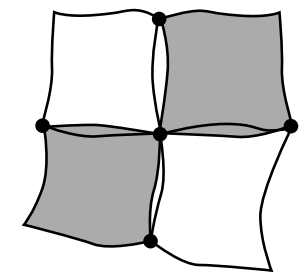

(b)

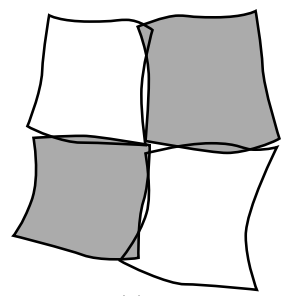

(c)

Figure 1. A bidimensional substructuring into subdomains and interfaces (a), and typical solutions before convergence for: FETI-DP (b), micro-macro $(c)$ 


\subsection{Modular treatment induced by substructuring}

Indeed, a substructuring allows to use different treatments on different subdomains. Their coupling has to be expressed on their common interface. This feature has been used for different kinds of couplings, for instance with an explicit/implicit/ modal dynamical treatment of different structural parts (Gravouil et al., 2001; Faucher et al., 2004), different spatial discretizations (Bernardi et al., 1994), for modal synthesis (Farhat et al., 1992; Rixen et al., 1998), or for different physics as for aeroelasticity, (Felippa et al., 2001).

In this section, we are more concerned with the purely structural problems for which the fields on the interface are (for quasi-statics): the displacement field $\underline{V}$, the force field $\underline{F}$. A representation on two scales may consists in splitting additively these fields, on each interface independently, (Ladevèze et al., 1999): $\underline{V}=\underline{V}^{m}+\underline{V}^{M}$ and $\underline{F}=\underline{F}^{m}+\underline{F}^{M}$. Superscript $M$ denotes the macro part, while superscript $m$ denotes the complementary micro part. Note that an additional condition on this splitting has to be prescribed to uniquely defined each contribution (a kind of orthogonality between macro and micro spaces) and to make it worth.

\subsection{Convergence acceleration using a coarse space}

Today, the most efficient domain decomposition methods embed a coarse problem, global to the whole domain, to improve their convergence, and to make them few sensitive to the increase of the number of subdomains (and therefore to the increase in the discretized problem size). This property is known as the numerical scalability (Bramble et al., 1986).

Let us first discuss this property for the FETI-DP method (Farhat et al., 2001). This approach first selects the so called 'corner' nodes, shared between several subdomains, Figure 1 (b), and avoiding any floating subdomain (with rigid body motions) if clamped. The total displacement field is prescribed to be continuous at each of these nodes, leading to a coarse problem whose degrees of freedom are the 'corner' displacement unknowns. Lagrange multipliers are used to glue the displacements on interfaces (on interface nodes other than 'corner' ones), and are solved iteratively. This approach proved to be numerically scalable for 2D and plate problems, but not for 3D massive problems. To recover numerical scalability in this case, an augmentation of the algorithm is proposed (Farhat et al., 2000b) which is close to a splitting into macro and micro quantities on the interfaces. The residual at a given iteration is a displacement gap on the interface; the proposed augmentation is the requirement to get a null average of the gap on each interface independently, Figure 2. If one selects the macro space on an interface as this average value, the augmentation is interpreted as the macro displacement continuity at each iteration: $\underline{V}^{M}=\underline{V}^{M^{\prime}}$ (on a local interface between two substructures, superscript $M$ denotes the macro part on a first side of the interface, i.e. for the first substructure, and superscript $M^{\prime}$ denotes the macro part on the second side of the interface, i.e. for the other substructure). This constraint is 
prescribed by using a Lagrange multiplier which can itself be interpreted as the macro force (i.e. the resultant of gluing forces on the interface): $\lambda=\underline{F}^{M}=-\underline{F}^{M^{\prime}}$. This multiplier is an additional unknown that increases the coarse problem size. Nevertheless, the extensibility is recovered and the resulting algorithm is more efficient with respect to the CPU usage.
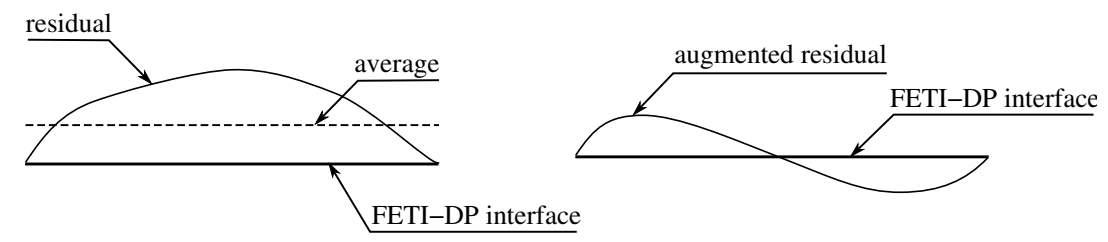

Figure 2. FETI-DP residual evolution on an interface

\subsection{Multilevel domain decomposition and homogenization}

Other multilevel representations can be used. One can choose the macro part of an interface field as the generalized averages of this field (Ladevèze et al., 1999): for instance, the already mentioned classical average (i.e. the constant part of the field) which corresponds to translations for the displacement, and to the resultant for the force. The linear part of the interface fields then corresponds to rotations, extensions and distortion in the plane of the interface for the displacement $\underline{V}$ and to torques and membrane forces for the forces $\underline{F}$. The degree of these generalized averages is limited to 1 (i.e. the linear part), Figure 3, which is usually a good compromise between convergence rate and cost.

As before, the continuity of macro displacements on each interface $\underline{V}^{M}=\underline{V}^{M^{\prime}}$, and equilibrium of macro forces $\underline{F}^{M}+\underline{F}^{M^{\prime}}=0$, are enforced at each iteration. To get a unique splitting into macro and micro parts, the 'orthogonality'

$$
\int_{\Gamma_{E E^{\prime}}} \underline{F}^{M} \cdot \underline{V}^{m} d S=0=\int_{\Gamma_{E E^{\prime}}} \underline{F}^{m} \cdot \underline{V}^{M} d S
$$

is used, and the macro part of the unknowns constitutes the degrees of freedom of the coarse problem, Figure 1 (c).

With this micro-macro representation, the macroscopic behaviour of a subdomain (the homogenized behaviour of the corresponding elementary representative volume) is the relationship between a macro force distribution on all of its interfaces, and the macro part of the corresponding displacement trace on the same interfaces. Using this homogenized stiffness on the left hand side of the coarse space leads to a multilevel domain decomposition with an improved convergence rate.

This approach has been used in particular for heterogeneous continuum media (Ladevèze et al., 2001), multi-cracked structures (Nouy et al., 2002; Ladevèze et 
al., 2003), non smooth discrete media (Nineb et al., 2007), and composite structures modelled up to a micro scale (Lubineau et al., 2006; Violeau et al., 2006).
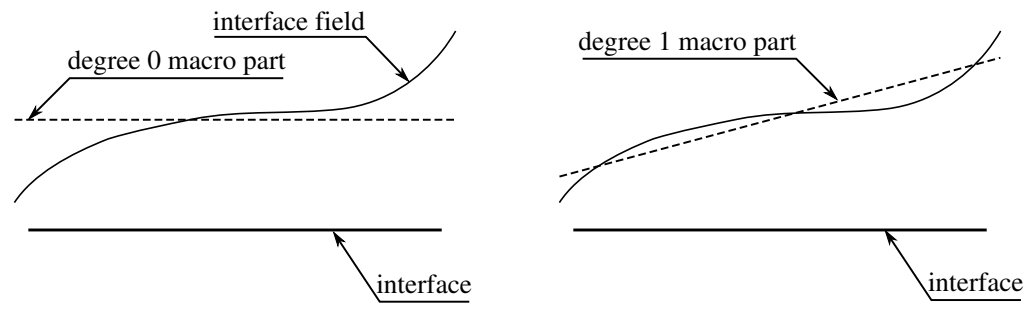

Figure 3. Micro-macro field evolution on an interface

\subsection{Structural zooming}

The previous micro-macro description can also lead to a gluing technique between subdomains described with different refinement levels corresponding to different spatial scales. The scale coupling is therefore performed on interfaces. The direct application is a structural zooming technique, allowing for the fine discretization of interest areas only (i.e. of particular subdomains only). With a LATIN solver, such an application is developed in (Guidault et al., 2006; Guidault et al., 2007). For plate problems, with a FETI-DP like solver, it is presented in (Amini et al., 2006).

Let us also mention that couplings between different discretizations or different models in subareas can also be performed with overlapping subdomain approaches. For instance, the Arlequin framework (Ben Dhia, 1998; Ben Dhia et al., 2005) and the approach detailed in next Section, can be seen as belonging to this category.

\section{Field transfer between non matching discretizations for multiphysics problems}

We are concerned herein with multiphysics problems which are strongly coupled in the body. In such cases, the different physics interact at the constitutive relations level. One of the simplest cases is saturated poroelasticity (Lewis et al., 1998; Coussy, 2004); the macroscopic constitutive relations are:

$$
\boldsymbol{\sigma}=\boldsymbol{D} \boldsymbol{\varepsilon}-b p \mathbf{1} \quad q=\frac{1}{Q} \dot{p}+b \operatorname{Tr} \dot{\varepsilon} \quad \underline{W}=H \underline{Z}
$$

The structure-related fields are: the stress field $\sigma$ and the strain field $\varepsilon$. The fluidrelated fields are: the accumulation rate of fluid $q$ and the opposite of Darcy velocity $\underline{W}$, the pore pressure field $p$ and its gradient $\underline{Z}$. Finally, $\boldsymbol{D}$ is Hooke tensor, $b$ and $Q$ are Biot coefficient and modulus, and $H$ is the permeability. 
The admissibility of the different fields concerns the conservation of momentum and mass. They lead to two decoupled systems of equations, one for the structure part, the other one for the fluid part. The corresponding variational formulations are:

- for the structure: $\underline{U} \in \mathcal{U}, \varepsilon=\frac{1}{2}\left(\operatorname{Grad} \underline{U}+\operatorname{Grad} \underline{U}^{T}\right)$, and

$$
\forall \underline{U}^{\star} \in \mathcal{U}_{0}, \int_{\Omega} \operatorname{Tr}\left[\boldsymbol{\sigma} \varepsilon\left(\underline{U}^{\star}\right)\right] d \Omega=\int_{\partial_{2} \Omega} \underline{F}_{d} \cdot \underline{U}^{\star} d S
$$

- for the fluid: $p \in \mathcal{P}, \underline{Z}=\underline{\operatorname{grad}} p$, and

$$
\forall p^{\star} \in \mathcal{P}_{0}, \int_{\Omega}\left(q p^{\star}+\underline{W} \cdot \underline{\operatorname{grad}} p^{\star}\right) d \Omega=\int_{\partial_{4} \Omega} w_{d} p^{\star} d S
$$

$\mathcal{U}$ (respectively $\mathcal{P}$ ) is the set of finite energy displacement (respectively pore pressure) fields, satisfying to the boundary condition $\left.\underline{U}\right|_{\partial_{1} \Omega}=\underline{U}_{d}$ (respectively $\left.p\right|_{\partial_{3} \Omega}=p_{d}$ ). $\partial_{1} \Omega$ is a first part of the boundary $\partial \Omega ; \underline{F}_{d}$ is a prescribed external force on the complementary part $\partial_{2} \Omega$. $\partial_{3} \Omega$ is another part of the boundary and $w_{d}$ is a prescribed fluid flux on the complementary part $\partial_{4} \Omega$. $\mathcal{U}_{0}$ and $\mathcal{P}_{0}$ are the corresponding sets with homogeneous boundary conditions.

These equations are two linear and decoupled problems for the structure and the fluid parts of the unknowns.

Several solving strategies can be used for the reference problem [2]-[4] (together with an appropriate initial condition). A partitioning strategy will avoid a coupled treatment of both the structure and the fluid (Felippa et al., 2001), for instance, an iterative strategy that will solve on one hand the local but coupled constitutive equations [2], and on the other hand the decoupled but global admissibility conditions [3],[4]. The LATIN (LArge Time INcrement) approach (Ladevèze, 1999) dedicated to multiphysics problems can be used in such a way; more details on this approach can be found in (Dureisseix et al., 2003b; Dureisseix et al., 2003a). There are also connections with the NSCD (Non Smooth Contact Dynamic) approach, for which the interactions took place between rigid grains; indeed, this approach also focuses on the contact / friction interactions, while the admissibility equations are the non smooth dynamics of the grains (Moreau, 1999; Jean, 1999).

An advantage of the partitioning approaches lies in their modularity. We will illustrate this point while considering the management of different discretizations for each physics.

\subsection{Using spatial non matching meshes}

Let us consider the case of a porous ceramic filter. The fluid to filter goes through the multi-perforated block of Figure 4 (upper right), and a pressure increase (up to 
a stabilized value) is prescribed for the inflow, while the outflow relative pressure is null.
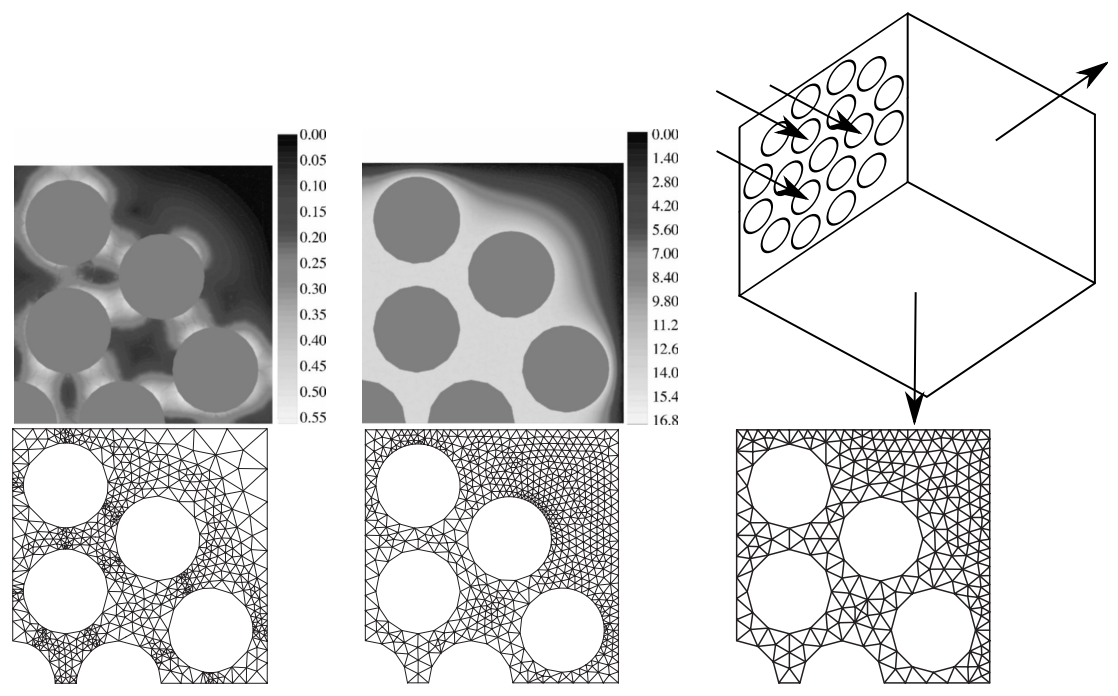

Figure 4. Principal strain field (upper left), pore pressure field (upper middle), inflow and outflow (upper right), and structure, fluid and interface meshes (lower)

The problem is modeled with the assumption of 2D plane strain and the symmetries are used to limit the study to a quarter of the section on Figure 4. The reported principal strain field and pore pressure field are obtained at the last time step of the transient simulation. This case exemplifies the interest to provide different meshes for each physics, as those of Figure 4 (bottom), since the gradients in the solution are not obtained at the same place for the different physics. A tool to transfer the fields from one discretization to an other, during the iterations of the solving procedure, is therefore required.

Such a tool has been firstly designed for thermo-viscoelasticity coupled problems, solved with a partitioning strategy relying on a fixed point method (Dureisseix et $a l .$, 2006). It is re-used here for poroelasticity. The field to transfer (stress, strain...) is usually available at integration points of a first mesh $\Omega_{1}$. The first step consists in its extrapolation to the nodes of each finite element independently; the resulting field, denoted with $E_{1}$ can be interpolated, and is a priori discontinuous throughout the element edges. To project $E_{1}$ into a field $E_{2}$ defined on a second mesh $\Omega_{2}$, a discontinuous mortar-like technique is used: generalized averages of the field are preserved, with respect to discontinuous test functions $F_{2}^{\star}$ on the target mesh $\Omega_{2}$ :

$$
\forall F_{2}^{\star}, \quad \int_{\Omega_{2}} E_{2} \cdot F_{2}^{\star} d \Omega=\int_{\Omega_{1}} E_{1} \cdot F_{2}^{\star} d \Omega
$$

As test functions, we propose to use the restriction to each element of the finite element basis functions, with an interpolation degree selected according to the field to be 


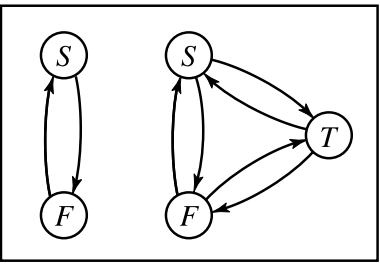

(a)

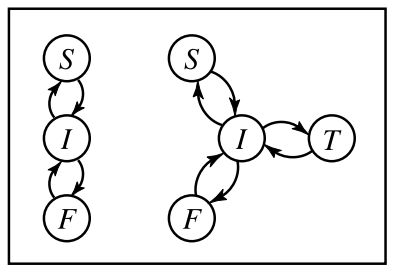

(b)

Figure 5. Modifications of transfer operations when adding a new physics; (a): without an interface mesh, $(b)$ : with such a mesh

transferred; for instance, if a quadratic interpolation of displacement is used, a linear test function is used for strain and stress; if a linear interpolation of pore pressure is used, the same linear test functions are used for the pressure and the fluid accumulation rate, etc. Finally, the projected field $E_{2}$ is interpolated to the integration points of $\Omega_{2}$, for each element independently.

The backward transfer from $\Omega_{2}$ to $\Omega_{1}$ is the dual operation, i.e. the transposed projector with respect to the energy norm $\int_{\Omega} F \cdot E d \Omega$. This formulation is symmetric: the same couple of dual projector is obtained if one begins with transferring fields from $\Omega_{2}$ to $\Omega_{1}$.

The solver used herein for the poroelastic problem is the LATIN method (Ladevèze, 1999). Basically, it solves iteratively on one hand the admissible conditions [3] and [4] separately on the different meshes of Figure 4, and on the other hand, the coupled constitutive relations [2]. We chose here to use a third mesh at each integration point of which the last problem is solved independently. This mesh is called the 'interface' mesh, Figure 4. The use of such a mesh allows more flexibility when adding new coupled physics. This is illustrated on Figure 5 when a third physics is added; $S$ stands for solid, $F$ for fluid, $T$ for thermal problem, for instance, and $I$ for the interface dedicated discretization.

\subsection{Using non-matching time discretizations}

For the time multiscale aspect, the same poroelastic problem will serve as an illustration. In order to focus on the time evolution of the solution, let us consider a pure strain monodimensional consolidation problem. The constitutive relations [2] read:

$$
\sigma=E \varepsilon-b p \quad q=\frac{1}{Q} \dot{p}+b \dot{\varepsilon} \quad W=H Z
$$

where $E$ is Young modulus. The admissibility equations [3],[4] lead to

$$
\frac{\partial \sigma}{\partial x}=0 \quad q=\frac{\partial W}{\partial x} \quad Z=\frac{\partial p}{\partial x}
$$


where $x \in[0, L]$ is the spatial coordinate. With a prescribed traction $\sigma_{d}(t)$ at $x=L$, the problem reads:

$$
\underbrace{\left[\begin{array}{cc}
-E & b \\
b & 1 / Q
\end{array}\right]}_{\boldsymbol{M}} \frac{d}{d t}\left[\begin{array}{l}
\varepsilon \\
p
\end{array}\right]-\underbrace{\left[\begin{array}{cc}
0 & 0 \\
0 & H
\end{array}\right]}_{\boldsymbol{A}} \frac{\partial^{2}}{\partial x^{2}}\left[\begin{array}{l}
\varepsilon \\
p
\end{array}\right]=\left[\begin{array}{c}
-\dot{\sigma}_{d} \\
0
\end{array}\right]
$$

If $\lambda_{i}$ and $V_{i}$ are the generalized eigenvalues and eigenvectors of $\boldsymbol{A} V_{i}=\lambda_{i} \boldsymbol{M}_{i} V_{i}$ (with a regular $M$ ), the corresponding homogeneous system leads to decoupled scalar equations $\dot{y}_{i}-\lambda_{i} \partial^{2} y_{i} / \partial x^{2}=0$. For $\lambda_{i}>0$, the solution is

$$
y_{i}=e^{-t / \tau_{i}}\left(A_{i} \cos \frac{x}{\sqrt{\lambda_{i} \tau_{i}}}+B_{i} \sin \frac{x}{\sqrt{\lambda_{i} \tau_{i}}}\right)
$$

To exhibit the characteristic times $\tau_{i}$, boundary conditions are required. If the observation scale is the same for all the physics, and if $L$ is the characteristic spatial length of the phenomenon that one wishes to capture, null boundary conditions for $x=0$ and $x=L$ lead to $A_{i}=0$ and the first non zero root of $\sin \left(L / \sqrt{\lambda_{i} \tau_{i}}\right)=0$ is $\tau_{i}=\left(1 / \lambda_{i}\right)(L / \pi)^{2}$. For the proposed example, one obtains $\lambda_{1}=0$ for the structure (since no viscosity is involved, the characteristic time for the structure is given by the external load, not by the physics). For the fluid, one gets:

$$
\tau_{2}=\frac{1}{H}\left(\frac{1}{Q}+\frac{b^{2}}{E}\right)\left(\frac{L}{\pi}\right)^{2}
$$

For the problems treated in (Néron et al., 2008), a ratio of 4 between the characteristic times are obtained ( $0.167 \mathrm{~s}$ for the loading on the solid, $\tau_{2}=0.043 \mathrm{~s}$ for the fluid). For aeroelasticity problems, a ratio of 10 to 20 is often obtained. Therefore, using different time discretizations is of interest, for instance to get a solution with an iso-quality for each of the physics (i.e. the same discretization error for the pressure, with respect to the exact pressure, and for the strain, with respect to the exact strain).

Several strategies are available to deal with a problem coupling different time grids. For instance, with an incremental approach, a time marching strategy similar to (Rey et al., 2005; Farhat et al., 2000a; Combescure et al., 2003; Faucher et al., 2003) is possible. When relying on the LATIN method as a solver, the situation is different since it is not an incremental approach (Ladevèze, 1999). Indeed, at each iteration, the solution is provided on the whole domain and on the whole studied time interval. If each physics has its own time discretization, this coupling also occurs on the interface' between the physics when solving the coupled constitutive relations. Therefore, this 'interface' is also equipped with its own time discretization, and a transfer operator between different time grids is also required. The same tool as for the spatial transfer can be re-used, provided that the time representation allows discontinuities between time slabs (similar to spatial discontinuity through finite element edges), and that the time integration scheme relies on a variational formulation. This is the case for the time discontinuous Galerkin approach (Eriksson et al., 1985; Borri et al., 1993). 
This representation has been used in (Néron et al., 2008) to provide a transfer operator between different time grids for coupled poroelastic problems (for the fluid, the structure and the interface). Coupling both time and spatial different discretizations within the same simulation is the next application and is currently under development.

\section{Conclusions}

Tools for field transfers between non matching discretizations have been presented (both for time or space). They allow to perform iterative resolutions where different scales interfer. Flexibility of such approaches is largely due to the use of an interface between the fields represented at different scales. This interface can be a geometric interface between subdomains, or a more numerical one between different grids or between different physics. In each case, these interfaces possess their own behavior and discretization. This framework could also be used for the case of model coupling. Among dedicated application cases, one can find: the coupling and transition between a discrete representation (for instance with discrete element models) and a continuous one, for fragmentation; the multiphysics coupling as in granular / fluid interaction for flow through divided media; the mechanical / convection-diffusion coupling for modeling hygromechanical phenomena, or biomechanical ones such as bone remodelling.

Acknowledgements

For the first part of this article, the developed tools arise from a collaboration with P. Ladevèze (LMT-Cachan) and P. Alart (LMGC); for the second one, with H. Bavestrello (Stanford University) and D. Néron (LMT-Cachan).

\section{References}

Amini A. M., Dureisseix D., Cartraud P., Buannic N., “ A micro-macro strategy for ship structural analysis with FETI-DP method”, Third European Conference on Computational Mechanics - ECCM 2006 - Solids, Structures and Coupled Problems in Engineering, 2006.

Ben Dhia H., " Multiscale mechanical problems: the Arlequin method", Comptes Rendus à l'Académie des Sciences, vol. 326, n³26, p. 899-904, 1998. Série IIb.

Ben Dhia H., Rateau G., “ The Arlequin method as a flexible engineering design tool”, International Journal for Numerical Methods in Engineering, vol. 62, p. 1442-1462, 2005.

Bernardi C., Maday Y., Patera A. T., “ A new nonconforming approach to domain decomposition: the mortar element method", in H. Brezzi (ed.), Nonlinear partial differential equations and their applications, Paris, p. 13-51, 1994.

Borri M., Bottaso C., “ A general framework for interpreting time finite element formulations”, Computational Mechanics, vol. 13, p. 133-142, 1993.

Bramble J. H., Pasciak J. E., Schatz A. H., “ The construction of preconditioners for elliptic problems by substructuring, I”, Mathematics of Computation, vol. 47, n 175, p. 103-134, 1986. 
Combescure A., Gravouil A., Herry B., " An algorithm to solve transient structural nonlinear problems for non-matching time-space domains", Computers and Structures, vol. 81, p. $1211-1222,2003$.

Coussy O., Poromechanics, John Wiley \& Sons, 2004.

Dureisseix D., Bavestrello H., “ Information transfer between incompatible finite element meshes: application to coupled thermo-viscoelasticity", Computer Methods in Applied Mechanics and Engineering, vol. 195, n 44-47, p. 6523-6541, 2006.

Dureisseix D., Ladevèze P., Néron D., Schrefler B. A., “ A multi-time-scale strategy for multiphysics problems: application to poroelasticity”, International Journal for Multiscale Computational Engineering, vol. 1, n 4, p. 387-400, 2003a.

Dureisseix D., Ladevèze P., Schrefler B. A., “ A LATIN computational strategy for multiphysics problems: application to poroelasticity", International Journal for Numerical Methods in Engineering, vol. 56, n 10, p. 1489-1510, 2003 b.

Eriksson K., Johnson C., Thomée V., “Time discretization of parabolic problems by the discontinuous Galerkin formulation”, RAIRO Modélisation Mathématique et Analyse Numérique, vol. 19, p. 611-643, 1985.

Farhat C., Géradin M., “ Using a reduced number of Lagrange multipliers for assembling parallel incomplete field finite element approximations", Computer Methods in Applied Mechanics and Engineering, vol. 97, p. 333-354, 1992.

Farhat C., Lesoinne M., “ Two efficient staggered algorithms for the serial and parallel solution of three-dimensional nonlinear transient aeroelastic problems", Computer Methods in Applied Mechanics and Engineering, vol. 182, p. 499-515, 2000a.

Farhat C., Lesoinne M., Le Tallec P., Pierson K., Rixen D., “ FETI-DP: a dual-primal unified FETI method - part I: a faster alternative to the two-level FETI method", International Journal for Numerical Methods in Engineering, vol. 50, n 7, p. 1523-1544, 2001.

Farhat C., Lesoinne M., Pierson K., “ A scalable dual-primal domain decomposition method”, Numerical Linear Algebra with Applications, vol. 7, p. 687-714, 2000 b.

Farhat C., Roux F.-X., “ Implicit parallel processing in structural mechanics”, in J. T. Oden (ed.), Computational Mechanics Advances, vol. 2, North-Holland, 1994.

Faucher V., Combescure A., “ A time and space mortar method for coupling linear modal subdomains and non-linear subdomains in explicit structural dynamics", Computer Methods in Applied Mechanics and Engineering, vol. 192, n 5-6, p. 509-533, 2003.

Faucher V., Combescure A., " Local modal reduction in explicit dynamics with domain decomposition. Part 2: specific interface treatment when modal subdomains are involved", International Journal for Numerical Methods in Engineering, vol. 61, n 1, p. 69-95, 2004.

Felippa C. A., Park K. C., Farhat C., " Partitioned analysis of coupled mechanical systems", Computer Methods in Applied Mechanics and Engineering, vol. 190, p. 3247-3270, 2001.

Gravouil A., Combescure A., “ Multi-time-step explicit-implicit method for non-linear structural dynamics”, International Journal for Numerical Methods in Engineering, vol. 50, p. $199-225,2001$.

Guidault P.-A., Allix O., Champaney L., Cornuault C., “ Une approche micro-macro pour le suivi de fissure avec enrichissement local", Revue Européenne de Mécanique Numérique, vol. 15, p. 187-198, 2006. 
Guidault P.-A., Allix O., Navarro J.-P., " A two-scale approach with homogenization for the computation of cracked structures", Computers and Structures, vol. 85, n 17-18, p. 13601371, 2007.

Jean M., “ The non-smooth contact dynamics method”, Computer Methods in Applied Mechanics and Engineering, vol. 177, p. 235-257, 1999.

Ladevèze P., Nonlinear computational structural mechanics - New approaches and nonincremental methods of calculation, Springer Verlag, 1999.

Ladevèze P., Dureisseix D., “ A new micro-macro computational strategy for structural analysis”, Comptes-Rendus de l'Académie des Sciences, vol. 327, p. 1237-1244, 1999.

Ladevèze P., Loiseau O., Dureisseix D., “ A micro-macro and parallel computational strategy for highly heterogeneous structures", International Journal for Numerical Methods in Engineering, vol. 52, $\mathrm{n}^{\circ} 1-2$, p. 121-138, 2001.

Ladevèze P., Nouy A., “ On a multiscale computational strategy with time and space homogenization for structural mechanics", Computer Methods in Applied Mechanics and Engineering, vol. 192, p. 3061-3087, 2003.

Le Tallec P., “ Domain decomposition methods in computational mechanics", Computational Mechanics Advances, vol. 1, North-Holland, 1994.

Lewis R. W., Schrefler B. A., The finite element method in the static and dynamic deformation and consolidation of porous media, 2nd edn, John Wiley \& Sons, 1998.

Lubineau G., Ladevèze P., Violeau D., “ Durability of CFRP laminates under thermomechanical loading: A micro-meso damage model”, Composites Science and Technology, vol. 66, p. 983-992, 2006.

Magoulès F. (ed.), Mesh partitioning techniques and domain decomposition methods, CivilComp Press / Saxe-Coburg, 2007.

Moreau J. J., “ Numerical aspects of sweeping process", Computer Methods in Applied Mechanics and Engineering, vol. 177, p. 329-349, 1999.

Néron D., Dureisseix D., “ A computational strategy for poroelastic problems with a time interface between coupled physics", International Journal for Numerical Methods in Engineering, 2008. To appear.

Nineb S., Alart P., Dureisseix D., “ Domain decomposition approach for nonsmooth discrete problems, example of a tensegrity structure”, Computers and Structures, vol. 85, $\mathrm{n}^{\circ} 9$, p. 499-511, 2007.

Nouy A., Ladevèze P., Loiseau O., “ A multiscale computational approach for contact problems", Computer Methods in Applied Mechanics and Engineering, vol. 191, p. 4869-4891, 2002.

Rey C., Chiaruttini V., “ An efficient multiscale parallel-in-time strategy for nonlinear poroplastic problems”, 8th ESAFORM Conference on Material Forming, p. 137-140, 2005.

Rixen D., Farhat C., Géradin M., " A two-step, two-field hybrid method for the static and dynamic analysis of substructure problems with conforming and non-conforming interfaces", Computer Methods in Applied Mechanics and Engineering, vol. 154, n 154, p. 229-264, 1998.

Violeau D., Ladevèze P., Lubineau G., “ Micromodel based computations for laminated composites", 8th International Conference on Computational Structures Technology, 2006. 